\section{FLAMENCO Y DERECHOS DE AUTOR. EL CASO DE CAMARÓN DE LA ISLA}

\author{
Jesús Heredia-Carroza \\ Universidad de Sevilla \\ ORCID iD: https://orcid.org/0000-0003-2280-2680 \\ jhercar12@gmail.com \\ Luis Palma Martos \\ Universidad de Sevilla \\ ORCID iD: https://orcid.org/0000-0001-5834-3629 \\ Ipalma@us.es \\ Luis F. Aguado \\ Pontificia Universidad Javeriana \\ ORCID iD: https://orcid.org/0000-0002-1675-0418 \\ Ifaguado@javerianacali.edu.co
}

Cómo citar este artículo/Citation: Heredia-Carroza, J., Palma Martos, L. y Aguado, L. F. (2019). Flamenco y derechos de autor. El caso de Camarón de la Isla. Arbor, 195 (791): a496. https:// doi.org/10.3989/arbor.2019.791n1009

Recibido: 14 febrero 2018. Aceptado: 24 julio 2018.

RESUMEN: En el artículo, utilizando elementos conceptuales de derecho comparado (originalidad) y de economía de la cultura (valor cultural), se expone que la contribución realizada por el intérprete a la obra flamenca no cuenta con una protección acorde con su importancia. La metodología utilizada incluye entrevistas a personalidades del flamenco, que permitieron determinar la estructura de la obra musical flamenca y el diseño de su proceso de creación. Posteriormente, a partir de encuestas, se obtiene una aproximación a la valoración cultural de los agentes que dan lugar a la obra: palo (acervo cultural), autor e intérprete. Conmemorando el 25 aniversario de su muerte, se presenta un caso representativo de la problemática tratada, protagonizado por Camarón de la Isla. En este sentido el trabajo contribuye al estudio de la valoración del intérprete como parte fundamental de la obra musical flamenca y, por tanto, sustenta la demanda de una protección por derechos de autor.

PALABRAS CLAVE: Flamenco; intérprete; valor cultural; derechos de autor; Camarón de la Isla.

\section{FLAMENCO AND COPYRIGHT. THE CASE OF CAMARÓN DE LA ISLA}

Copyright: (C) 2019 CSIC. Este es un artículo de acceso abierto distribuido bajo los términos de la licencia de uso y distribución Creative Commons Reconocimiento 4.0 Internacional (CC BY 4.0).

ABSTRACT: This article, through conceptual elements of Comparative Law (originality) and Cultural Economics (cultural value), analyzes the gap between the performer's contribution to the flamenco work and the protection he/she obtains. We designed a methodology based on interviews with flamenco personalities and surveys of flamenco market agents. By means of 15 interviews, the structure of the flamenco musical work and its creation process were determined and 690 surveys enabled us to obtain an approximation to the cultural valuation of the contribution of the three elements that constitute the flamenco work: palo (cultural heritage), author and performer. For the 25th anniversary of his death, we present a representative case focusing on Camarón de la Isla. The article contributes to analyzing the value of the performer as a key element of the flamenco musical work and, therefore, supports the claim for his/her protection by copyright.

KEYWORDS: Flamenco; performer; cultural value; copyright; Camarón de la Isla. 


\section{INTRODUCCIÓN}

El maestro salió del camerino, podía escuchar la agitación y la excitación que provenía del auditorio. Cuando se abrió la cortina y apareció en el escenario, el público guardó silencio, Camarón se sentó en su silla de anea y de repente surgió de su ser la soleá llamada salud es lo que yo quiero. El público estaba paralizado; cuando terminó el cantaor, se levantó para aplaudir en medio del éxtasis. No sabían que esta sería su última actuación, apenas seis meses después la vida del artista se extinguiría por causa del cáncer. Es en este momento cuando surge el dilema, tan pronto como él murió, su riqueza y ganancias murieron con él, dejando a su familia y sus futuras generaciones sin su sustento económico (Marcos, 2010, 24 de noviembre).

El artículo se centra en la figura de Camarón de la Isla, conmemorando el 25 aniversario de su desaparición. Camarón es considerado uno de los mejores cantaores de la historia del flamenco (Gamboa y Núñez, 2003, p. 93). Pocas semanas antes de su muerte, en su última entrevista televisada, hacía referencia a su situación acerca de los derechos de autor que no poseía sobre las obras en las que participó (Sáenz de Tejada, 1992, 2 de agosto). Con ello se plantea un problema de repercusiones legales -se cuestiona la validez de firmas reconocidas como autoras de canciones-, morales (Román Pérez, 2003, p. 233) -se reivindica una mayor protección del trabajo del intérprete creativo ${ }^{1}$ y culturales -se descubre el escaso interés oficial e industrial, que no popular, por el reparto de derechos de la obra de un artista reconocido como único (Connolly y Krueger, 2006, p. 34; Heredia-Carroza, Palma Martos y Aguado Quintero, 2017, p. 183; Towse, 2010, p. 306)-.

Para entender el caso de Camarón hay que contextualizarlo dentro del flamenco, género de música popular tradicional (Bermúdez y Pérez, 2009, p. 131; Tongeren, 2017, p. 180) originario de Andalucía, España (Infante, 1980, p. 31). Cuenta con un componente regional importante unido a dicha comunidad autónoma (Aoyama 2007, p. 104; Aoyama, 2009, p. 81; Cruces Roldán, 2001, p. 16), donde existen estilos por zonas, ciudades e incluso barrios (Manuel, 2010, p. 108; Tongeren, 2017, p. 169). Además, se caracteriza por una tradición oral que se refleja en elementos como la improvisación o como la intuición, y en su repertorio, a menudo nutrido por obras populares (Galacho Abolafio, 2014, p. 379; Steingress, 2007, p. 48). Los cantes o palos ${ }^{2}$ se conocen por las personas que los interpretan y le dan su estilo propio, desco- nociéndose en muchos casos su origen, de ahí que sea considerado como un género musical vivo (Bermúdez y Pérez, 2009, p. 131). Ejemplos significativos son: las seguiriyas de Antonio Mairena o las soleares de Tomás Pavón. Su importancia y reconocimiento se reflejan en su inscripción en la Lista Representativa Cultural Inmaterial de la Humanidad en 2010 (Palma, Palma, Rodríguez, Martín y Cascajo, 2017, p. 59).

El argumento principal del estudio es considerar que el intérprete desarrolla un trabajo creativo que representa un valor cultural (Icazuriaga, Cuadrado y Miquel, 2016, p. 10; Klamer, 2003, p. 15). No obstante, este valor añadido no tiene una traducción jurídica en derechos equivalente a su importancia (Osborne, 2017, p. 573; Román Pérez, 2003, p. 39; Towse, 2007, p. 746). En la práctica se les da un tratamiento de elemento rutinario (Caves, 2003, p. 73), como se podrá observar en el caso de Camarón de la Isla.

Para ello, en primer lugar, se analiza el texto refundido de la ley de propiedad intelectual (en adelante TRLPI), norma fundamental en este ámbito en el ordenamiento jurídico español, para luego comprobar los estándares de originalidad que utilizan otros estados europeos y los Estados Unidos para proteger el trabajo creativo. En segundo lugar, se presenta el proceso de creación de la obra musical flamenca, cuyos principales argumentos son: palo, autor e intérprete creativo. Conceptualmente se sustenta con la bibliografía existente (Felton, 1978, p. 43; Towse, 2007, p. 746) y con entrevistas realizadas a personalidades del mundo del flamenco ${ }^{3}$. En tercer lugar, se valora la figura del intérprete mediante encuestas realizadas a tres agentes del mercado del flamenco: aficionados, críticos y gestores de espacios culturales. A efectos del estudio, la muestra se divide en: aficionados ${ }^{4}$ y expertos ${ }^{5}$ (Lundy y Smith, 2016, p. 141). Por último, se presenta el caso de Camarón de la Isla, para lo cual, siguiendo el hilo argumental trazado en el artículo, se usa información de las entrevistas realizadas y se realiza un estudio del número de visitas de sus interpretaciones en la plataforma digital Youtube.

La contribución de la investigación es, por un lado, conceptual, con el análisis de los requisitos necesarios para proteger los trabajos creativos que forman la obra musical flamenca según diferentes ordenamientos jurídicos en materia de propiedad intelectual; por otro lado, es empírica, reflejando el desequilibrio entre el valor cultural aportado por el intérprete y la protección que recibe (Osborne, 2017, p. 573), ejemplificado con el caso concreto de Camarón de la Isla. 


\section{MARCO CONCEPTUAL}

2.1. Derechos de autor vs. derechos vecinos en la legislación española

Para estudiar el caso de Camarón de la Isla, es necesario analizar el TRLPI, donde se observa que la ubicación de los derechos de autor y de los intérpretes es diferente (Román Pérez, 2010). Por un lado, los primeros se recogen en el libro I (de los Derechos de autor), mientras que los de los intérpretes aparecen en el libro II (de los otros derechos de propiedad intelectual y de protección "sui generis" de las bases de datos, junto con otros titulares de derechos de propiedad intelectual, como los productores de fonogramas, audiovisuales o las entidades de radiodifusión).

Los derechos de autor (Plant, 1934, p. 26; Throsby, 2016, p. 155), recogidos en el libro I se centran en los derechos patrimoniales (Giblin, 2017, p. 184) -reproducción, distribución, comunicación pública y transformación- y los morales (Baldwin, 2014, p. 29; Rushton, 1998, p. 24) -paternidad, integridad, divulgación, modificación, retirada del comercio por cambio de convicciones intelectuales o morales y acceso a ejemplar único o raro-.

Los derechos vecinos, centrados en los sujetos que aparecen en el libro II, no incluyen el derecho patrimonial de transformación, ni los morales de divulgación, modificación, retirada del comercio por cambio de convicciones intelectuales o morales y acceso a ejemplar único o raro (Towse, 2007, p. 746).

Un tema controvertido es la duración de los derechos patrimoniales, ya que los derechos de los autores duran toda su vida y setenta años después de su muerte o declaración de fallecimiento, y sin embargo los de los artistas duran setenta años desde la interpretación (véase Directive 2011/77/EU of the European Parliament and of the Council of 27 September 2011, amending Directive 2006/116EC on the term of protection of copyright and certain related rights) o desde la divulgación lícita de la grabación de su ejecución.

La diferencia es sustancial en la duración (longitud) ${ }^{6}$ de los derechos de uno y otro (Towse, 2007, p. 751) además de su amplitud ${ }^{7}$ (cobertura), siendo en ambos casos minusvalorada la contribución del intérprete en términos de protección. La posible explicación es que los sujetos que aparecen en el libro II no aportan naturaleza creativa a la obra y lo que se pretende proteger es la inversión económica o de trabajo que realizan, dándoles tratamiento de hummdrum input a sus aportaciones (Caves, 2003, p. 73; Sánchez Aristi, 2017, p.1552).

En este sentido, se deberían diferenciar en el TRLPI dos bloques: el de los que aportan altura creativa a la obra (autores e intérpretes) y el de los que participan con recursos no creativos, como los financieros o los que realizan la grabación de la obra (Hadida, 2010, p. 47). En el próximo apartado se tratarán los estándares de originalidad necesarios para que alguien pueda ser considerado autor de cualquier tipo de obra y, así, pueda acogerse a los derechos que aparecen en el libro I del TRLPI.

\subsection{La originalidad como requisito de protección por derechos de autor}

Para entender la protección de la creatividad o valor cultural es necesario definir el concepto de originalidad en sus vertientes subjetiva y objetiva (Galacho Abolafio, 2017, p. 1298; Heredia-Carroza et al., 2017, p. 175; Román Pérez, 2003, p. 39). Para ello, además del TRLPI y sentencias emitidas por el Tribunal Supremo español, se estudian regulaciones legales y jurisprudencia de diferentes países.

Por un lado, la vertiente subjetiva de la originalidad según Galacho Abolafio (2017, p. 1298) se basa en la impronta personal de los creadores. Esto se desprende de lo dictado por la Cour de Cassation ${ }^{8}$ francesa (caso Furtwängler), donde se observa la primacía de la impronta personal como criterio de originalidad por encima del de novedad (caso Manitas de Plata) ${ }^{9}$ (Heredia-Carroza et al., 2017, p. 182).

El artículo 2.2 de la ley de creaciones intelectuales personales individuales alemana (Persönliche Geistige Shöpfungen) equipara el concepto de originalidad subjetiva al de altura creativa (Gestaltungshöhe). A diferencia del tribunal francés, la regulación alemana demanda un nivel mínimo de novedad en la obra, siendo su estándar de originalidad el más exigente de Europa. Sin embargo, el reputado jurista alemán Loewenheim (Román Pérez, 2003, p. 20) trata conceptos como espíritu humano (der menschliche Geist), individualidad y expresión personal del creador, todos ellos muy ligados al de impronta personal definido anteriormente.

Por otro lado, la vertiente objetiva de la originalidad se basa, según lo dictado por el Tribunal Supremo ${ }^{10}$ español, en tres requisitos: singularidad, individualidad y distinguibilidad. Todos ellos fundamentados en el presupuesto primordial de que para que la creación 
humana sea protegida, esta debe ser original y haber creado algo que no existía anteriormente. Dichos requisitos están en consonancia con el concepto de variación distinguible (distinguishable variation) utilizado por los tribunales de Estados Unidos, según el cual una obra es original cuando las aportaciones realizadas por los creadores proporcionan una variación suficiente con respecto a la obra preexistente (Code of Federal Regulations, Title 37 - Patents, Trademarks, and Copyrights).

Por último, las Directivas Comunitarias ${ }^{11}$ concernientes a propiedad intelectual están alineadas con el criterio de originalidad objetiva, donde la novedad determina el reconocimiento como obra y la protección por derechos de autor que se atribuye a su creador, por encima del criterio de impronta personal sustentando por la originalidad subjetiva.

Una vez definido el concepto de originalidad desde el punto de vista jurídico, ahora se va a identificar con elementos que conforman el valor cultural aportado por los agentes que intervienen en la creación de la obra. Para ello se presenta el proceso de creación de la obra musical flamenca, donde se podrán observar los agentes intervinientes que agregan valor cultural y, por tanto, merecen una protección jurídica.

\section{PROCESO DE CREACIÓN DE LA OBRA MUSICAL FLAMENCA}

Gracias a la bibliografía existente (Romero, 1996, p. 75; Rosón, 2010, p. 29) y a las quince entrevistas realizadas a personalidades del flamenco (véase anexo 1) se ha podido diseñar el proceso de creación de la obra flamenca (véase anexo 2). Para ello, se han tenido en cuenta los agentes que intervienen en el mismo ${ }^{12}$-palo, autor e intérprete creativo- y los elementos aportados por cada uno de ellos. Dichos elementos se denominan elementos estructurales y de ellos se desprende la originalidad de la obra, que potencialmente podrá ser protegida por los derechos de propiedad intelectual. En la figura 1 se muestra dicho proceso y las aportaciones realizadas por los agentes intervinientes (Heredia-Carroza, Palma Martos y Aguado, 2019, p. 5).

En él se distinguen los agentes que intervienen en la creación de la obra:

Palo: aporta dos elementos estructurales que tienen origen en el acervo cultural flamenco: ritmo y armonía (Manuel, 2010, p. 111; Rosón, 2010, p. 32). Desde la perspectiva de la bibliografía sobre la propiedad intelectual está expuesto al dominio público ${ }^{13}$ (Galacho Abolafio, 2014, p. 349; Geiger, 2017, p. 92; Manuel, 2010, p. 119).

Figura 1. Proceso de creación de la obra musical flamenca

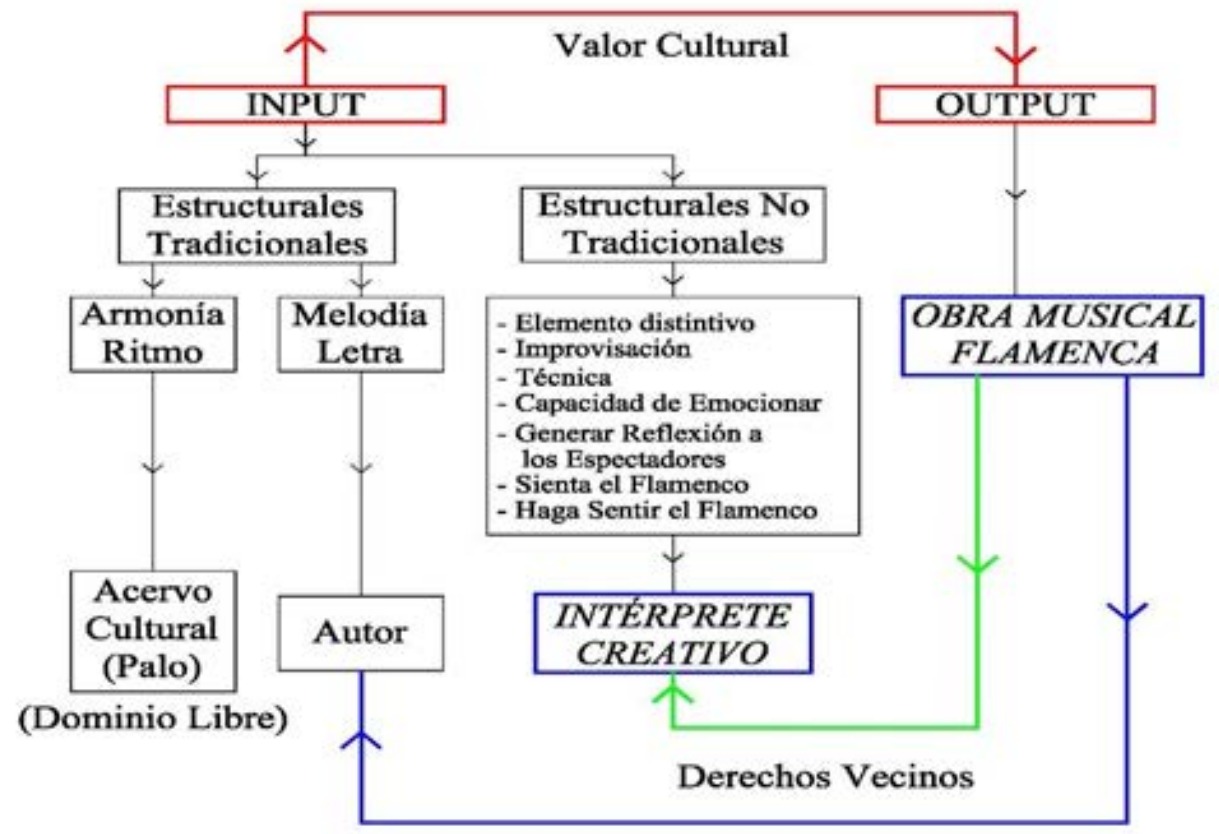

Derechos de Autor

Fuente: Elaboración propia. 
Autor: mediante la composición ${ }^{14}$ aporta dos elementos estructurales más a la obra: melodía y letra (Rosón, 2010, p. 30; Throsby, 1990, p. 69; Werck y Heyndels, 2007, p. 28). El instrumento de protección de este tipo de trabajo creativo son los derechos de autor (Manuel, 2006, p. 128).

Intérprete: mediante la exteriorización de la obra, aporta variables identificadas en las entrevistas como son: elemento distintivo, improvisación, técnica, capacidad de emocionar, generar reflexión en los espectadores, que el receptor sienta y haga sentir el flamenco, entre otras (Hadida, 2010, p. 50; Levinson, 2015, p. 76; Towse, 2007, p. 748). El instrumento de protección de este tipo de trabajo creativo son los derechos vecinos.

Este trabajo se alinea con la postura de considerar la interpretación creativa como un quinto elemento estructural (Heredia-Carroza et al., 2017, p. 177). Al ser la música un producto intangible, necesita en su proceso de creación la exteriorización para ser un producto completo, ya que pocas personas pueden traducir lo escrito en partituras en sonidos (Felton, 1978, p. 43; Sánchez Aristi, 2017, p. 1565). Además, en el caso que nos ocupa del flamenco, durante la exteriorización el intérprete recrea lo preestablecido por el autor en la composición, lo que puede considerarse como un trabajo creativo con entidad propia. De esta forma se podría llegar a hacer equivalentes la creatividad del autor y el talento del intérprete (Bryant y Throsby, 2006, p. 522), siguiendo la tesis marcada por la profesora Towse (2007). Gráficamente la importancia de la exteriorización se recoge en la Figura 2.

Figura 2. Secuencias del proceso de creación

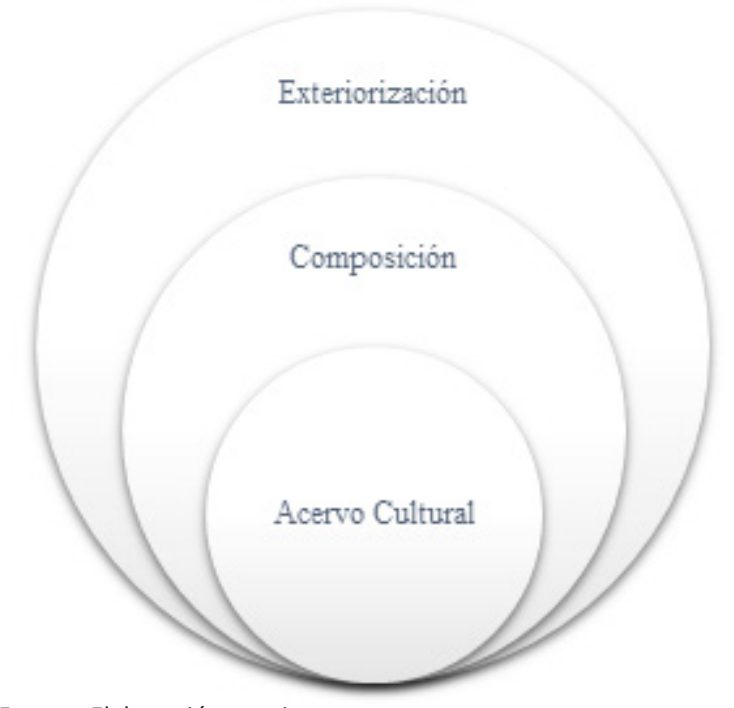

Fuente: Elaboración propia.
Otra idea relacionada con el proceso de creación son los denominados por Landes y Posner (1989, p. 344) costes de expresión. Mientras que el trabajo creativo del autor queda cerrado cuando termina de componer la melodía y de escribir la letra, la aportación creativa del intérprete no finaliza con su primera interpretación. Debido a las particularidades interpretativas del flamenco, es fundamental la constante recreación de la obra por parte de los intérpretes (Ordóñez Flores, 2011, p. 19). Dicho esfuerzo intelectual no es tenido en cuenta por los derechos de propiedad intelectual.

El proceso de creación presentado puede aplicarse a otros tipos de música popular donde durante la exteriorización se recree lo preestablecido por la composición, como son la salsa, el jazz o el blues (Negus, Street y Behr, 2017, p. 375). Además de las personas entrevistadas, Galacho Abolafio (2014, p. 353) habla del carácter improvisatorio, esencial para dichos estilos, lo que está unido a la importancia de las contribuciones realizadas por el intérprete, que hacen que la percepción del valor cultural de la obra varíe y la obra sea única (Hernández Belver y Ullán de la Fuente, 1996, p. 52; Galacho Abolafio, 2017, p. 1301).

\section{EL INTÉRPRETE FLAMENCO DESDE LA PERSPECTIVA DE AFICIONADOS Y EXPERTOS}

En este apartado se pretenden contrastar las ideas explicadas anteriormente sobre la importancia del intérprete con el sentir de los agentes del mercado del flamenco. Para ello se han realizado encuestas a través de un instrumento en línea dispuesto en Flama. La Guía del Flamenco, que además contó con la divulgación del Centro Andaluz de Documentación del Flamenco y la web Promocionmusical.es. En total se realizaron 690 encuestas, 580 a aficionados, 51 a críticos y 59 a gestores culturales ${ }^{15}$ en el periodo comprendido entre mayo y julio de 2017.

Se han agrupado en dos categorías: 110 expertos y 580 aficionados al flamenco (Lundy y Smith, 2016, p. 141). Bajo la categoría de expertos se enmarcan los críticos encargados de valorar el flamenco en prensa o en concursos y los gestores culturales que evalúan las obras para su programación en espectáculos. Ambas figuras poseen un alto nivel de capital cultural que les permite realizar juicios por medio de la crítica y la contratación de un espectáculo (Bourdieu, 1984, p. 66).

Se diseñaron dos instrumentos de encuesta, cada uno dirigido a una categoría de valoración (Lundy y Smith, 2016, p. 141). El instrumento se dividió en cua- 
tro apartados, siendo los apartados 2, 3 y 4 comunes para ambos. En el primer apartado, para los expertos, se indagó en el perfil profesional (años en el ejercicio de crítica y en el medio de publicación de las críticas; número de años en la gestión y la importancia relativa del flamenco en la programación general del espacio cultural). Para los aficionados, en este apartado se investigaron sus características y patrones de consumo (grado de conocimiento del flamenco, si asiste a eventos, con qué frecuencia..., entre otros). En el segundo, se realizaron 41 preguntas de valoración de la obra musical flamenca. En el tercer apartado, se exploró sobre política cultural, con cuatro preguntas acerca de si el flamenco se debe apoyar con fondos públicos para su promoción. En el cuarto apartado se pregunta por los aspectos socio-demográficos básicos (por ejemplo, edad, sexo, nivel educativo...).

Para los efectos de este artículo, se han tomado las respuestas acerca de la figura del intérprete. La primera de ellas está relacionada con el nivel de aportación que realiza el intérprete a la obra musical flamenca [desde 1 hasta 7, siendo 1 muy bajo y 7 muy alto]. Para interpretar el resultado también se ha agrupado la valoración en los siguientes tres niveles: bajo [1 y 2]; medio [3, 4 y 5]; alto [6 y 7] y no sabe/no contesta.

Como se puede observar en la figura 3 , tanto expertos como aficionados se decantan en una amplia mayoría por darle un valor alto al papel desarrollado por el intérprete dentro de la obra musical flamenca. Esto se debe a ideas que se han expuesto anteriormente como son: la oralidad del género, la importancia de la exteriorización de la obra y el repertorio nutrido de obras populares. En última instancia es el intérprete el que materializa la obra, dotándola de sus características propias como son la técnica, la improvisación o la manera de sentir.

La importancia del intérprete también se puede observar en la valoración de las siguientes afirmaciones $^{16}$, recogidas en la figura 4 , donde tanto aficionados como expertos han valorado su grado de acuerdo:

De dichas afirmaciones y su valoración se desprende que:

Aficionados y expertos consideran que el éxito o fracaso de la obra depende en gran medida del intérprete.

La elección de asistencia a un concierto también depende en gran medida del intérprete.

Consideran esencial la aportación del intérprete a la obra musical flamenca.

Las tres cuestiones tienen relación directa con los gustos y preferencias de aficionados y expertos (Miquel-Romero y Montoro-Pons 2017, p. 7; Towse, 2016, p. 141). Con las dos primeras afirmaciones se pone de manifiesto que el elemento diferencial (Bourdieu, 1984 , p. 66) que los hace decantarse a asistir o no a un espectáculo es el intérprete, y con la última se enfatiza que su aportación es esencial para la obra musical.

Figura 3. Valoración del intérprete

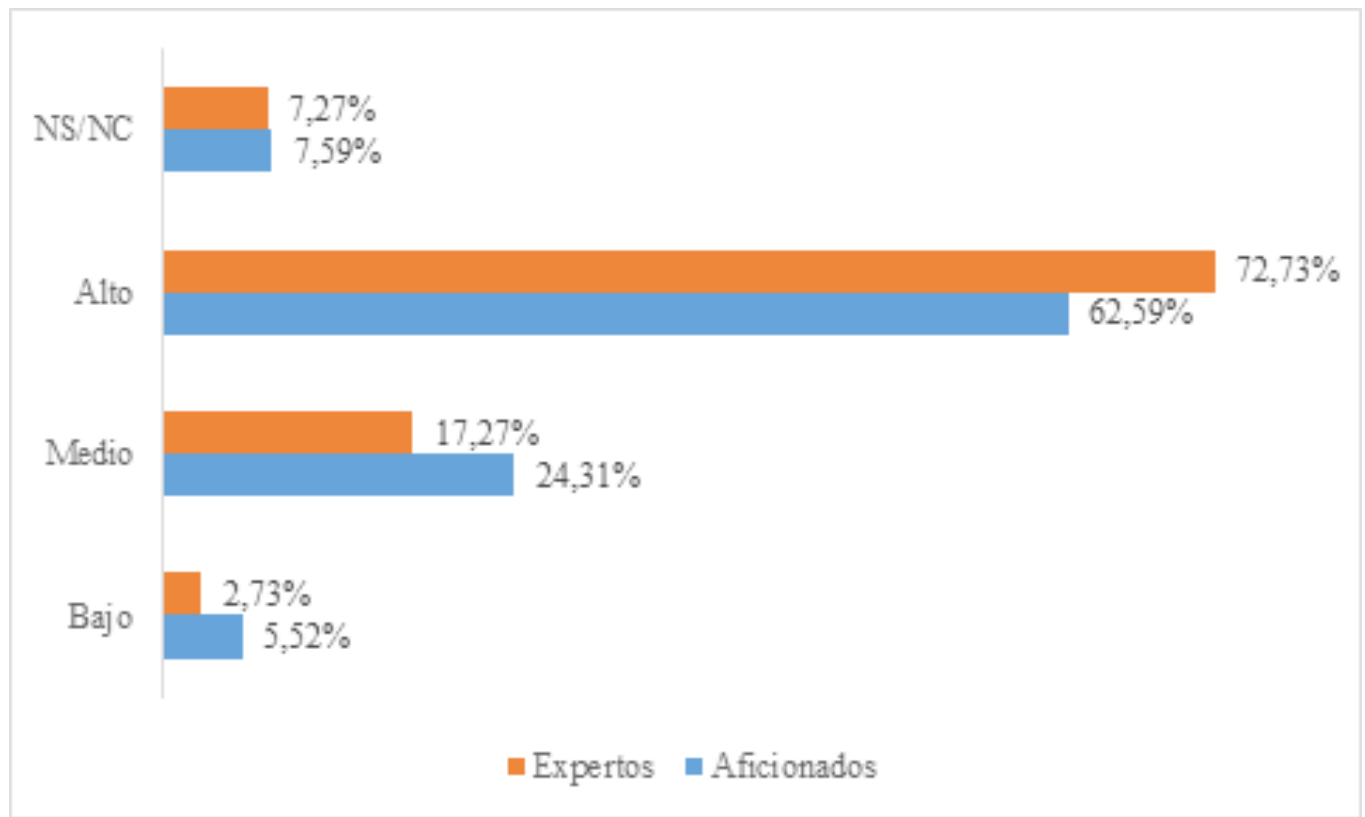

Fuente: Elaboración propia. 
Figura 4. Valoración de características del intérprete (Af.: aficionado / Exp.: experto)

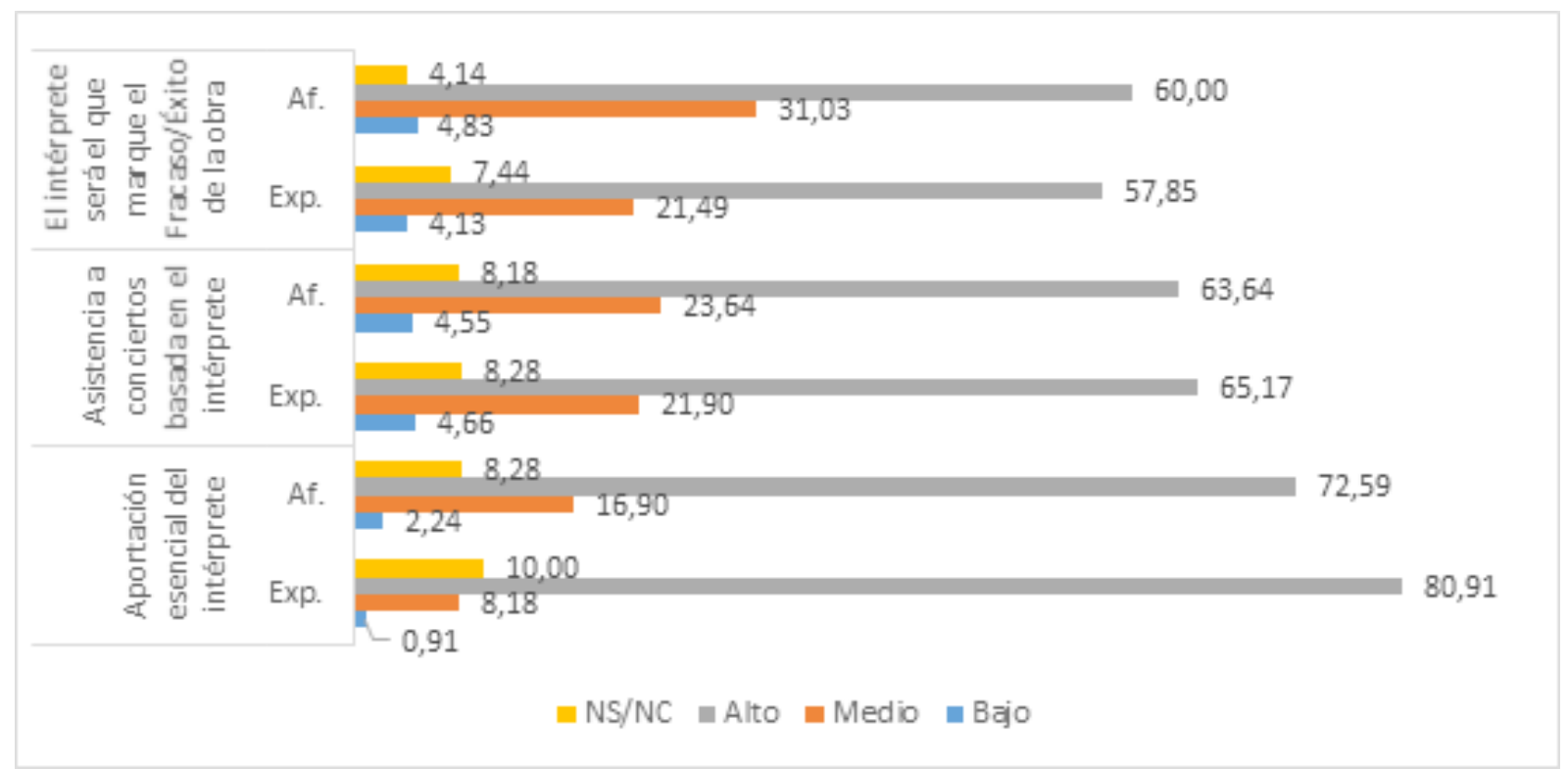

Fuente: Elaboración propia.

Todo ello influye en la consolidación de la marca propia del artista (Hernando y Campo, 2017), que actúa como un elemento que acentúa la diferenciación de los bienes culturales e influye en la decisión de compra del consumidor (Monasterio Astobiza, 2017, p. 6; Yoo y Donthu, 2001, p. 1). El intérprete actúa como un elemento que reduce la incertidumbre del aficionado acerca de las características de la obra, haciendo que se decante por una u otra dependiendo de la persona que la interprete (Heredia-Carroza et al., 2017, p. 181; Zieba y O'Hagan, 2010, p. 148).

No obstante, los diferentes ordenamientos jurídicos en materia de propiedad intelectual no dan al intérprete una protección acorde con la importancia que tiene para el mercado. Como se ha podido comprobar, para ambas categorías definidas las aportaciones realizadas por el intérprete son fundamentales para la obra musical flamenca (Negus et al., 2017, p. 375). El caso de Camarón de la Isla es un ejemplo notable de cómo el reconocimiento dado por el mercado no se traduce en una mayor protección jurídica.

\section{CAMARÓN DE LA ISLA}

Una vez se han explicado tanto la parte jurídica como la identificación y valoración de las aportaciones realizadas por el intérprete creativo, se procede a presentar el caso de Camarón de la Isla, dando de esta forma un ejemplo visible que sustenta la argumentación presentada durante el desarrollo del trabajo.

Camarón de la Isla es considerado uno de los mejores cantaores de la historia, fue un transgresor, que abrió nuevas vías de interpretación (Gamboa y Núñez, 2003, p. 93), reconocimiento que se puede observar en los diferentes actos que han conmemorado en el presente año el 25 aniversario de su muerte ${ }^{17}$. Quizás sea el caso más sonado y polémico que existe sobre derechos de autor en el flamenco. Esto es debido a las figuras que reúne; por un lado, la familia de Lucía ${ }^{18}$ y, por otro, la de Camarón de la Isla (Manuel, 2010, p. 125; Sáenz de Tejada, 1992, 2 de agosto), ambas consideradas muy importantes para el flamenco.

Las afirmaciones emitidas por las personalidades del flamenco acerca de Camarón son una de las aportaciones más interesantes de este artículo:

"El más grande de todos los tiempos" (Marina Heredia).

"El mejor de los completos" (Barullo), haciendo referencia a que normalmente un cantaor suele tener determinados palos en los que destaca, cuando para él Camarón destacaba en todos.

"Camarón era capaz de coger letras o recursos de otros cantaores, y engrandecerlos cuando los ponía en su voz" (Paco Cepero) ${ }^{19}$. 
“Camarón veía más allá, lo demostró con la leyenda del tiempo, muchos de los que estábamos allí no lo veíamos claro, sin embargo, él siempre creyó en que lo que se estaba haciendo era de calidad, como luego el tiempo demostró" (Tomatito) ${ }^{20}$.

"Sus recursos técnicos además de un gran estudio y conocimiento necesitaban de unos atributos físicos que solo él tenía" (Rocío Márquez).

"Fue el único flamenco de su época que fue capaz de mover a las masas y que cuando sacaba un disco fuera número uno en ventas" (José Mercé).

Estas afirmaciones, junto con la valoración emitida por los encuestados (110 expertos y 580 aficionados) sustentan la tesis de que sin la figura de Camarón la obra musical flamenca no tendría el mismo valor cultural y reconocimiento (Hutter y Frey, 2010, p. 37).

De la entrevista al guitarrista Tomatito se pudo extraer información sobre cómo trabajaron para la confección del álbum conceptual ${ }^{21}$ la leyenda del tiempo, obra de culto para el flamenco, que marcó un antes y un después para dicho género. El guitarrista contaba cómo dicho álbum salió al mercado gracias a la creencia de Camarón de poder convertir el flamenco en un género que llegara a las masas. Además, describió el proceso de creación de dicho trabajo, cómo las obras creadas por Kiko Veneno y Ricardo Pachón, entre otros, llegaban a manos de los intérpretes y luego estos añadían sus elementos propios. De esta forma, el valor cultural de la obra aumentaba, lo que confirma el esquema del proceso de creación explicado anteriormente.

El caso de Camarón es un ejemplo claro de intérprete creativo considerado una superestrella
(Meiseberg, 2014, p. 10; Pitt, 2010, p. 227). Debido a la imposibilidad de sustituir sus aportaciones por otras provenientes de otros artistas (Benhamou, 2016, p. 55; Connolly y Krueger, 2006, p. 34; Towse, 2010, p. 306), las obras que interpretó se consideran únicas, siendo Camarón el elemento que las hace reconocibles ante el público. Esto se puede demostrar, ya que las mismas obras interpretadas por otros artistas nunca han llegado a generar el mismo nivel de impacto (Cox, Felton y Chung, 1995, p. 334).

\subsection{Impacto de Camarón en Youtube}

Relacionada con la última idea expuesta, se presenta otra de las contribuciones del estudio: la utilización de Youtube (Bürkner y Lange, 2017, p. 34) para comprobar el nivel de impacto de determinadas obras cuando cambia el intérprete. Según las encuestas realizadas es el medio más común para escuchar flamenco por los aficionados ${ }^{22}$. El atractivo de comprobar el número de visitas obtenidas en dicha plataforma digital es demostrar la estabilidad temporal de las interpretaciones hechas por Camarón. En la época en la que Camarón actuaba no existía dicha plataforma y, sin embargo, sus interpretaciones reciben visitas después de 25 años de su muerte. Siguiendo la línea marcada por Simonton (2014, p. 20) se demuestra que dichas obras interpretadas por Camarón "sobreviven en el tiempo". Al cambiar el cantaor, el valor percibido de la obra varía, quedando patente que una misma obra interpretada por Camarón frente a otro intérprete tiene un mayor valor para el público, lo que se materializa en el mayor número de visitas y en la citada "supervivencia temporal". En la tabla 1 se presentan algunos casos significativos.

Tabla 1. Número de visitas en Youtube de las obras interpretadas por Camarón vs. otros intérpretes (agosto 2017)

\begin{tabular}{|c|c|c|}
\hline \multicolumn{1}{|c|}{ Obra } & Versión & No Visitas en Youtube \\
\hline \multirow{2}{*}{ Como el agua } & Camarón & 1.627 .854 \\
\cline { 2 - 3 } Volando voy & Pepe de Lucía & 2.599 .724 \\
\hline \multirow{2}{*}{ Soy gitano } & Camarón & 701.759 \\
\cline { 2 - 3 } & Kiko Veneno & 3.751 .311 \\
\cline { 2 - 3 } La leyenda del tiempo & Camarón & 213.976 \\
\cline { 2 - 3 } & Camarón & 854.890 \\
\cline { 2 - 3 } & Enrique Morente & 77.524 \\
\hline \multirow{2}{*}{ Viviré } & Camarón & 272.445 \\
\cline { 2 - 3 } & Paco de Lucía y Pepe de Lucía & 31.893 \\
\hline
\end{tabular}

Fuente: Youtube. 
Con los datos obtenidos por las encuestas y por el número de visitas en Youtube se puede fundamentar que la interpretación es la que hace decantarse al espectador por la escucha de una obra u otra. En este caso se puede observar cómo Camarón actúa como un elemento diferencial (Caves, 2003, p. 73) que mueve a los oyentes a escuchar su versión y no otra de las muchas ofrecidas por el mercado. En casos como este, la aportación de originalidad por parte del intérprete sirve para justificar la consideración de la interpretación como un trabajo creativo fundamental para la obra en música popular tradicional.

\section{CONCLUSIONES}

En el artículo se presenta una metodología para el flamenco, que puede ser aplicable a otros tipos de música popular tradicional que esté caracterizada por su oralidad y la importancia del intérprete en su proceso creativo. Esto permite la identificación de los agentes intervinientes y la posterior medición del valor cultural aportado por cada uno.

El intérprete con sus aportaciones imprime características distintivas a la obra musical flamenca que, una vez exteriorizada, es considerada como única. Dicha interpretación podría ser imitable, pero nunca podrá ser reproducible debido a las características únicas del intérprete que la realizó. Dichas características (improvisación, técnica o capacidad de emocionar) se pueden sintetizar en el concepto de originalidad.

Como se puede observar, la aplicación de los instrumentos actuales de protección del trabajo creativo en música popular tradicional (derechos de autor y derechos vecinos), crea distintos niveles de protección (Flegel y Roth, 2014, p. 1097; Weiner, 2004, p. 537), provocando un desequilibrio en el mercado (Heredia-Carroza et al., 2017, p. 178). Dicho desequilibrio afecta negativamente al intérprete, ya que los elementos aportados por él son protegidos por los derechos vecinos, que tienen una menor cobertura.

El resultado de las encuestas a aficionados y a expertos ratifica que el mercado identifica el valor cultural aportado por el intérprete. Como se ha comprobado con el caso de Camarón de la Isla, el interés hacia las obras por parte del mercado crece cuando un sujeto con alta creatividad individual o mucho talento las interpreta. Por todo ello, se necesita una solución para el desequilibrio existente entre la importancia que tienen las contribuciones del intérprete a la obra y la protección que reciben por parte de los derechos de propiedad intelectual. En el presente estudio se proponen tres:

Por un lado, gracias al estudio de derecho comparado, se podría aplicar la línea marcada por las joint works de la legislación norteamericana (véase Code of Federal Regulations, Title 37- Patents, Trademarks, and Copyrights) y las obras en colaboración defendidas en el TRLPI en su artículo 7.1. Siguiendo la argumentación del proceso de creación expuesto, los agentes intervinientes -palo, autor e intérprete- dan lugar a un trabajo con un resultado unitario que sería protegido por derechos de autor. En este caso los derechos de los intérpretes aumentarían debido a que serían considerados como autores en colaboración.

Debido a la oralidad que caracteriza al flamenco, donde elementos como la improvisación y la intuición del intérprete son fundamentales, la obra es continuamente recreada. Esto abriría la posibilidad de considerarla como una obra compuesta, idea expuesta en el artículo 9 del TRLPI (Galacho Abolafio, 2014, p. 388). Dicha obra se caracteriza por ser una obra nueva, que incorpora una composición preexistente sin la colaboración de su autor.

Otra posible solución, para el caso de España, sería la aplicación del artículo 132 del TRLPI (Román Pérez, 2010, p. 3). Según lo recogido en el mismo, subsidiariamente se pueden aplicar disposiciones propias del libro I (donde aparecen los autores) a los sujetos que aparecen en el libro II (como son los intérpretes). En este caso se homologarían los derechos entre autor e intérprete, pero con la diferencia de que no se consideraría al intérprete como autor de la obra final.

\section{AGRADECIMIENTOS}

Los autores agradecen a la abogada Ana Isabel Cejuela Solís y a la doctora Cristina Cruces Roldán los útiles comentarios y discusiones realizadas, así como a los expertos entrevistados, a las organizaciones que dieron a conocer la encuesta (Flama. La guía del flamenco, el Centro Andaluz de Documentación del Flamenco y el sitio web Promocionmusical.com) y a las personas que respondieron la encuesta.

El presente trabajo ha sido financiado por el Programa de Movilidad Académica entre Instituciones Asociadas de la Asociación Universitaria Iberoamericana de Posgrado (AUIP). 
1. El intérprete creativo es aquel que además de ejecutar la obra compuesta por el autor, es capaz de añadir elementos propios que actúen como elementos definitorios de la obra final que llega al mercado.

2. El palo es cada una de las variedades tradicionales del cante flamenco, como son: la seguiriya, la soleá, las bulerías o el fandango, entre otras. Estas variedades se distinguen por tener una métrica propia, denominada compás y una armonía delimitada para el acompañamiento (Manuel, 2010, p. 111).

3. Se realizaron entrevistas en profundidad a personalidades del flamenco y de la cultura en general. Se incluye a intérpretes, autores, gestores de espacios culturales o representantes de las sociedades de gestión de derechos. El criterio de selección de los entrevistados fue su relevancia en el mundo del flamenco y de la cultura (por ejemplo, artistas flamencos que hayan obtenido reputados premios nacionales e internacionales, gestores que dirijan importantes festivales y espacios culturales (como la Bienal de Flamenco de Sevilla). En el Anexo 1 se presenta el listado respectivo y la característica distintiva de cada entrevistado.

4. Por aficionado de flamenco se entiende aquella persona que guiada por sus preferencias está dispuesta a invertir recursos (tiempo, dinero) a través de su participación en espectáculos en vivo y grabaciones (Miquel-Romero y Montoro-Pons, 2017, p. 7; Montoro-Pons, Cuadrado García y Casaús-Estellés, 2013).

5. Por experto se entiende aquella persona que a través de un entrenamiento especializado y su experiencia acumulada (Holbrook, 1999, p. 144), ya sea mediante el estudio o el ejercicio de su profesión, cuenta con un alto grado de conocimiento artístico (Bourdieu, 1984, p. 66).

6. Según Varian (2005, p. 126$)$, el término longitud se refiere a la dimensión de la

\section{BIBLIOGRAFÍA}

Aoyama, Y. (2007). The role of consumption and globalization in a cultural industry: The case of flamenco. Geoforum, 38 (1), pp. 103-113. https://doi.org/10.1016/j. geoforum.2006.07.004

Aoyama, Y. (2009). Artist, Tourists, and the State: Cultural Tourism and the Flamen- propiedad intelectual que marca el final de los derechos.

7. Varian (2005, p. 125) define amplitud como el nivel de cobertura que ofrecen los derechos.

8. Sentencia de 4 de enero de 1964 , donde se estipula que se produce un "atentado al derecho del artista sobre la obra que constituye su interpretación" (Román Pérez, 2003, p. 167).

9. Sentencia de la Cour de Cassation de 1 de julio de 1970 (Román Pérez, 2003, p. 71).

10. Sentencia núm. 253/2017 del Tribunal Supremo, Sala 1. ${ }^{a}$, de lo Civil, 26 de abril de 2017 y Sentencia núm. 542/2004 del Tribunal Supremo, Sala 1.․ 24 de junio de 2004.

11. Directiva 2001/29/CE, de 22 de mayo - LCEur \2001\2153. Derecho de autor. Armonización de determinados aspectos de los derechos de autor y derechos afines a los derechos de autor en la sociedad de la información y Directiva 2011/77/UE del Parlamento Europeo y del Consejo de 27 de septiembre de 2011 por la que se modifica la Directiva 2006/116/CE relativa al plazo de protección del derecho de autor y de determinados derechos afines. Diario Oficial de la Unión Europea, 10 de octubre de 2011.

12. Para la creación de la obra hacen falta, por un lado, los recursos inmateriales (acervo cultural y las contribuciones artísticas del autor y del intérprete) y, por otro, los recursos financieros y materiales (equipos de grabación, instrumentos musicales, recursos económicos, etc.). El presente trabajo se ha centrado en las contribuciones inmateriales dependientes de la creatividad de las personas implicadas en la composición y exteriorización de la obra (Throsby, 2006, p. 2).

13. La expresión dominio público hace referencia a aquel patrimonio intelectual

co Industry in Andalusia, Spain. International Journal of Urban and Regional Research, 33 (1), pp. 80-104. https://doi. org/10.1111/j.1468-2427.2009.00846.x

Baldwin, P. (2014). The Battle between Anglo-American Copyright and European Authors' Rights. En Baldwin, P. que está libre de toda exclusividad en su acceso y utilización (Landes y Posner, 1989, p. 344)

14. Es de especial relevancia que el art. 10.1 TRLPI en su punto b), destinado a fijar el objeto de propiedad intelectual, trate el concepto de "composiciones musicales", no de "obras musicales", obviándose la etapa de exteriorización de la obra que, como se verá más adelante, es protegida mediante derechos vecinos.

15. Los cuestionarios y la base de datos están disponibles solicitándolos a los autores.

16. En el anexo 3 se adjuntan las preguntas completas.

17. Se han realizado actos como la declaración de 2017 como año Camarón en su ciudad natal, San Fernando (Cádiz). Se ha creado la producción Camarón Revolution, las exposiciones 25 años sin Camarón. Leyenda infinita y Camarón vive. Además, se ha organizado el Congreso Leyenda Camarón. 25 años, celebrado en octubre de 2017 en San Fernando (Cádiz). Se han realizado homenajes por la geografía española (San Fernando, Barcelona, Madrid, entre otras ciudades).

18. Formada por Paco de Lucía, Pepe de Lucía y el padre de ambos, Antonio Sánchez.

19. Guitarrista acompañante de Camarón en los primeros discos que grabó.

20. Tomatito fue el guitarrista que acompañó a Camarón de la Isla en su última etapa.

21. Con su publicación se rompe con la concepción de discos tradicionales de flamenco, pasando de realizar cantes a interpretar canciones.

22. De 580 consumidores encuestados, el $31,55 \%$ asegura que el medio que más utilizan para escuchar flamenco es dicha plataforma. En el anexo 4 se adjuntan los datos obtenidos en las encuestas.

(ed.). The Copyright Wars. Princeton University Press, pp. 14-53. https://doi. org/10.2307/j.ctt6wq0z2.4

Benhamou, F. (2016). Artists' labour markets. En Rizzo, I. y Towse, R. (eds). The Artful Economist. A new look at Cultural Economics. Springer, pp. 53-58. 
Bermúdez S. y Pérez, J. (2009). Introduction: Spanish Popular Music Studies. Journal of Spanish Cultural Studies, 10 (2), pp. 127-133. https://doi. org/10.1080/14636200902990661

Bourdieu, P. (1984). Distinction. Cambridge, MA: Harvard University Press.

Bryant, W. D. A. y Throsby, D. (2006). Creativity and the behavior of artists. En Ginsburg, V. A. y Throsby, D. (eds.) Handbook of the Economics of Art and Culture. Elsevier, pp. 507-530. https://doi. org/10.1016/S1574-0676(06)01016-7

Bürkner, H. J. y Lange, B. (2017). Sonic capital and independent urban music production: Analysing value creation and 'trial and error' in the digital age. City, Culture and Society, 10, pp. 33-40. https://doi. org/10.1016/j.ccs.2017.04.002

Caves, R. (2003) Contracts between art and commerce. The Journal of Economic Perspectives, 17 (2), pp. 73-84. https:// doi.org/10.1257/089533003765888430

Connolly, M. y Krueger A. B. (2006). Rockonomics: The Economics of Popular Music. En Ginsburg, V. A. y Throsby, D. (eds.) Handbook of the Economics of Art and Culture. Elsevier, pp. 667-719. https://doi. org/10.1016/S1574-0676(06)01020-9

Cox, R. K., Felton, J. M. y Chung, K. H. (1995). The concentration of Commercial Success in Popular Music: An analysis of the Distribution of Gold Records. Journal of Cultural Economics, 19 (4), pp. 333-340. https://doi.org/10.1007/BF01073995

Cruces Roldán, C. (2001). El flamenco como Patrimonio. Anotaciones a la Declaración de la voz de la Niña de los Peines como bien de interés cultural. Sevilla: Bienal de Arte Flamenco.

Felton, M. V. (1978). The economics of the creative arts: the case of the composer. Journal of Cultural Economics, 2 (1), pp. 41-61. https://doi.org/10.1007/BF02479731

Flegel, M. y Roth, J. (2014). Legitimacy, Validity, and Writing for Free: Fan Fiction, Gender, and the Limits of (Unpaid) Creative Labor. The Journal of Popular Culture, 47 (6), pp. 1092-1108. https://doi. org/10.1111/jpcu.12198

Galacho Abolafio, A. F. (2014). La obra derivada musical. Entre el plagio y los derechos de autor. Madrid: Aranzadi.

Galacho Abolafio, A. F. (2017). Artículo 105. Definición de artistas intérpretes o ejecutantes. En Palau Ramírez, F. y Palao Moreno, G. (dirs.) Comentarios a la Ley de Propiedad Intelectual. Valencia: Tirant lo Blanch, pp. 1291-1306

Gamboa, J. M. y Núñez, F. (2003). Camarón. Vida y obra. Iberautor Promociones Culturales.

Geiger, C. (2017). Copyright as an access right: Securing cultural participation through the protection of creators' interests. En Giblin, R. y Kimberlee, W. (eds.). What if we could reimagine copyright? Australian National University Press, pp. 73-109. https://doi. org/10.22459/WIWCRC.01.2017.03

Giblin, R. (2017). Reimagining copyright's duration. En Giblin, R. y Kimberlee, W. (eds.). What if we could reimagine copyright? Australian National University Press, pp. 177-211. https://doi. org/10.22459/WIWCRC.01.2017.06

Hadida, A. L. (2010) Commercial success and artistic recognition of motion picture projects. Journal of Cultural Economics, 34 (1), pp. 45-80. https://doi. org/10.1007/s10824-009-9109-z

Heredia-Carroza, J., Palma Martos, L. A. y Aguado Quintero, L. F. (2017). Originalidad subjetiva y copyright. El caso del flamenco en España. Anduli. Revista Andaluza de Ciencias Sociales, 16, pp. 175-194.

Heredia-Carroza, J., Palma Martos, L. y Aguado, L. F. (2019). Song, performance and authorship. The case of flamenco in Spain. Trames. A Journal of the Humanities and Social Sciences, 23 (1), pp. 3-14.

Hernández Belver, M. y Ullán de la Fuente, A. M. (1996). Estudio del comportamiento artístico desde la perspectiva psicosocial. El enfoque de Vigotsky. Arte, individuo y sociedad, 8, pp. 52-64.

Hernando, E. y Campo, S. (2017). Does the Artist's Name Influence the Perceived Value of an Art Work? International Journal of Arts Management, 19 (2), pp. 49-58.

Holbrook, M. B. (1999). Popular Appeal versus Expert Judgments of Motion Pictures. Journal of Consumer Research, 26. (2), pp. 144-155. https://doi.org/10.1086/209556

Hutter, M. y Frey, B. (2010). On the Influence of Cultural Value on Economic Value. Revue d'Economie Politique, 120 (1), pp. 35-46. https://doi.org/10.3917/redp.201.0035

Icazuriaga, J., Cuadrado, M. y Miquel, M. J. (2016). Analysing Perceived Value, Satisfaction and Purchase Intention in the Music Industry. $7^{\text {th }}$ Vienna Music Business Research Days. University of Music and Performing Arts Vienna, Austria.
Infante, B. (1980). Orígenes de lo flamenco y secreto del cante jondo. Sevilla: Consejería de Cultura. Junta de Andalucía.

Klamer, A. (2003). A pragmatic view on values in economics. Journal of Economic Methodology, 10 (2), pp. 191-212. https://doi. org/10.1080/1350178032000071075

Landes, W. M. y Posner, R. A. (1989). An Economic Analysis of Copyright Law. Journal of Legal Studies, 325 (33), pp. 344353. https://doi.org/10.1086/468150

Levinson, J. (2015). Musical Concerns, Essays in Philosophy of Music. Oxford: Oxford University Press. https://doi.org/10.1093/ acprof:oso/9780199669660.001.0001

Lundy, D. E. y Smith, J. L. (2016). It's Tough to be a Critic: Professional vs. Non-professional Music Judgment. Empirical Studies of the Arts, 35 (2), pp. 139-168. https:// doi.org/10.1177/0276237416661989

Manuel, P. (2006). The Saga of a Song: Authorship and Ownership in the Case of "Guantanamera". Latin American Music Review, 27 (2), pp. 121-147. https://doi. org/10.1353/lat.2007.0007

Manuel, P. (2010). Composition, Authorship and Ownership in Flamenco, Past and Present. Ethnomusicology, 54 (1), pp. 106-135. https://doi.org/10.5406/ ethnomusicology.54.1.0106

Marcos, C. (2010, 24 de noviembre). El último concierto de Camarón. El País. Disponible en https://elpais.com/diario/2010/11/24/ madrid/1290601469_850215.html

Meiseberg, B. (2014). Trust the artist versus trust the tale: performance implications of talent and self-marketing in folk music. Journal of Cultural Economics, 38 (1), pp. 9-42. https://doi.org/10.1007/ s10824-012-9196-0

Miquel-Romero, M. J. y Montoro-Pons, J. (2017). Consumption habits, perception and positioning of content-access devices in recorded music. International Journal of Arts Management, 19 (3), pp. 4-18.

Monasterio Astobiza, A. (2017). ¿Qué es cultura en la "economía de la cultura"? Definiendo la cultura para crear modelos mensurables en economía cultural. Arbor, 193 (783): 376. https://doi. org/10.3989/arbor.2017.783n1007

Montoro-Pons, J., Cuadrado García, M. y Casaús-Estellés, T. (2013). Analysing the popular music audience: determinants of participation and frequency of attendance. International Journal of Music Business Research, 2 (1), pp. 35-61. 
Negus, K., Street, J. y Behr, A. (2017). Copying, copyright and originality: imitation, transformation and popular musicians. European Journal of Cultural Studies, 20 (4), pp. 363-380. https://doi. org/10.1177/1367549417718206

Ordóñez Flores, E. (2011). La perpetua reinvención de la identidad de los géneros en el baile flamenco. Arte, individuo y sociedad, 23 (1), pp. 19-28. https://doi. org/10.5209/rev_ARIS.2011.v23.n1.2

Osborne, R. (2017). Is equitable remuneration equitable? Performers' rights in the UK. Popular Music and Society, 40 (5), pp. 573-591. https://doi.org/10.1080/0 3007766.2017.1348660

Palma, L., Palma, M. L., Rodríguez, A., Martín, J. L. y Cascajo, I. (2017). Live Flamenco in Spain: A Dynamic Analysis of Supply, with managerial implications. International Journal of Arts Management, 19 (3), pp. 58-70.

Pitt, I. L. (2010). Superstars effects on royalty income in a performing rights organization. Journal of Cultural Economics, 34 (3), pp. 219-236. https://doi. org/10.1007/s10824-010-9123-1

Plant A. (1934). The economic aspect of copyright in books. Economica, 1 (2), pp. 167195. https://doi.org/10.2307/2548748

Prieto-Rodríguez, J. y Fernández-Blanco, V. (2000). Are popular and classical music listeners the same people? Journal of Cultural Economics, 24 (2), pp. 147-164. https:// doi.org/10.1023/A:1007620605785

Román Pérez, R. E. de (2003) Obras musicales, compositores, intérpretes y nuevas tecnologías. Madrid: Reus.

Román Pérez, R. de (2010). Derecho de propiedad intelectual de los artistas intérpretes o ejecutantes como derecho análogo al derecho de autor. Diario La Ley, 7387.

Romero, J. (1996). La otra historia del Flamenco. Sevilla: Junta de Andalucía.

Rosón, T. (2010). El flamenco como obra musical, coreográfica y escénica. Las obras originales y derivadas: versiones, arreglos y utilización de las obras. En Castilla Barea, M. (coord.). El flamenco y los derechos de autor. Madrid: Reus, pp. 23-38.

Rushton, M. (1998). The moral Rights of Artists: Droit moral ou droit pécuniaire? Journal of Cultural Economics, 22 (1), pp. 15-32. https://doi. org/10.1023/A:1007454719802
Sáenz de Tejada, I. (1992, 2 de agosto). La herencia de un príncipe. El País. Disponible en: http://elpais.com/diario/1992/08/02/ cultura/712706402_850215.html

Sánchez Aristi, R. (2017). Comentario al artículo 105. En Bércovitz Rodríguez-Cano, R. (coord.). Comentarios a la Ley de Propiedad Intelectual. Madrid: Tecnos, pp. 1545-1575.

Simonton, D. K. (2014). Creative Genius in Literature, Music and the Visual Arts. En Ginsburg, V. A. y Throsby, D. (eds.) Handbook of the Economics of Art and Culture. Elsevier, pp. 14-49. https:// doi.org/10.1016/B978-0-444-537768.00002-7

Steingress, G. (2007). Flamenco postmoderno entre tradición y heterodoxia: un diagnóstico sociomusicológico (escritos 1989-2006). Sevilla: Signatura.

Throsby, C. D. (1990). Perception of quality in demand for the theatre. Journal of Cultural Economics, 14 (1), pp. 65-82. https://doi.org/10.1007/BF02268198

Throsby, D. (2006). An Artistic Production Function: Theory and Application to Australian Visual Artists. Journal of Cultural Economics, 30 (1), pp. 1-14. https://doi. org/10.1007/s10824-005-9001-4

Throsby, D. (2016). The Composer in the Market Place Revisited: The Economics of Music Composition Today. En Rizzo, I y Towse, R. The Artful Economist. A new look at Cultural Economics, Springer, pp. 153-170. https://doi.org/10.1007/9783-319-40637-4_9

Tongeren, C. van (2017). Distinctive culture: Framing flamenco artistry in Polígono Sur: El arte de las Tres Mil by Dominique Abel. Journal of Spanish Cultural Studies, 18 (2), pp. 169-189. https://doi.org/ 10.1080/14636204.2017.1308633

Towse, R. (2007). The Singer or the Song? Developments in Performers' Rights from the Perspective of a Cultural Economist. Review of Law and Economics, 3 (3), pp. 745-766. https://doi. org/10.2202/1555-5879.1158

Towse, R. (2010). A textbook of cultural economics. Cambridge: Cambridge University Press. https://doi.org/10.1017/ CBO9780511992131

Towse, R. (2016). Copyright and Music Publishing in the UK. En Rizzo, Iy Towse, R. The Artful Economist. A new look at Cultural Economics, Springer, pp. 133-152. https:// doi.org/10.1007/978-3-319-40637-4_8
Varian, H. R. (2005). Copying and Copyright. Journal of Economic Perspectives, 19 (2), pp. 121-138. https://doi. org/10.1257/0895330054048768

Weiner, R. G. (2004). Copyrights and Copywrong: The Rise of Intellectual Property and How It Threatens Creativity. The Journal of Popular Culture, 37 (3), pp. 536-539. https://doi.org/10.1111/ j.0022-3840.2004.084_4.x

Werck, K. y Heyndels, B. (2007). Programmatic choices and the demand for theatre: The case of Flemish theatres. Journal of Cultural Economics, 31 (1), pp. 25-41. https://doi.org/10.1007/s10824006-9026-3

Yoo, B. y Donthu, N. (2001) Developing and validating a multidimensional consumer-based brand equity scale. Journal of Business Research, 52 (1), pp. 1-14. https://doi.org/10.1016/S01482963(99)00098-3

Zieba, M. y O'Hagan, H. (2010). Output Characteristics and Other Determinants of Theatre Attendance: An Econometric Analysis of German Data. Applied Economics Quarterly, 56 (2), pp. 147-174. https://doi.org/10.3790/aeq.56.2.147

\section{Textos legales}

Real Decreto Legislativo 1/1996, de 12 de abril, por el que se aprueba el texto refundido de la Ley de Propiedad Intelectual, regularizando, aclarando y armonizando las disposiciones legales vigentes sobre la materia. Disponible en: http:// noticias.juridicas.com/base_datos/Ad$\mathrm{min} /$ rdleg1-1996.html

\section{Otros recursos}

Code of Federal Regulations, Title 37Patents, Trademarks, and Copyrights. Copyright Office regulations codified in the Code of Federal Regulations (CFR). These regulations are also known as Circular 96. Disponible en https://www. copyright.gov/title37/202/

Directive 2011/77/EU of the European Parliament and of the Council of 27 September 2011, amending Directive 2006/116EC on the term of protection of copyright and certain related rights. Disponible en: https://eur-lex.europa. eu/LexUriServ/LexUriServ.do?uri=OJ:L: 2011:265:0001:0005:EN:PDF 
Anexo 1. Composición del panel de expertos entrevistados

\begin{tabular}{|c|c|c|}
\hline \multirow{6}{*}{ Autores e intérpretes ${ }^{1}$} & $\begin{array}{l}\text { José Soto Soto } \\
\text { (José Mercé) }\end{array}$ & $\begin{array}{l}\text { Premio Masters of Mediterranean Music del } \\
\text { Mediterranean Music Institute (MMI) de Berklee College } \\
\text { of Music. Medalla de Andalucía } 2010.1 \text { disco doble } \\
\text { platino, } 2 \text { discos de platino y } 2 \text { discos de oro. }\end{array}$ \\
\hline & $\begin{array}{l}\text { José Fernández Torres } \\
\text { (Tomatito) }\end{array}$ & Medalla de oro al Mérito en las Bellas Artes³ 2016. \\
\hline & $\begin{array}{l}\text { Marina Heredia Ríos } \\
\text { (Marina Heredia) }\end{array}$ & $\begin{array}{l}\text { Giraldillo }{ }^{4} \text { al cante } 2016 \text {. Premio Mejor disco de cante } \\
\text { flamenco } 2010 \text {, por la crítica nacional de flamenco. }\end{array}$ \\
\hline & $\begin{array}{l}\text { Francisco López-Cepero } \\
\text { García } \\
\text { (Paco Cepero) }\end{array}$ & Medalla de oro al Mérito en las Bellas Artes 2003. \\
\hline & $\begin{array}{l}\text { Dra. Rocío Márquez Limón } \\
\text { (Rocío Márquez) }\end{array}$ & $\begin{array}{l}\text { Doctora por la Universidad de Sevilla en Estudios } \\
\text { avanzados de flamenco. Ganadora de la lámpara minera } \\
\text { en } 2008 \text { y del Giraldillo a la innovación } 2016 .\end{array}$ \\
\hline & $\begin{array}{l}\text { Juan Fernández Montoya } \\
\text { (Barullo) }\end{array}$ & $\begin{array}{l}\text { Ganador del Concurso Nacional de Córdoba }{ }^{6} 2016 . \\
\text { Modalidad Baile. }\end{array}$ \\
\hline Político e investigador & $\begin{array}{l}\text { Dr. Juan Manuel Suárez } \\
\text { Japón }\end{array}$ & $\begin{array}{l}\text { Consejero de Cultura y Medioambiente de la Junta de } \\
\text { Andalucía en el periodo 1990-1994. Catedrático de } \\
\text { Geografía Humana en la Universidad Pablo de Olavide. }\end{array}$ \\
\hline \multirow{2}{*}{$\begin{array}{l}\text { Representantes de la Sociedad } \\
\text { General de Autores y Editores } \\
\text { de España (SGAE) }\end{array}$} & D. Javier Losada Calvo & $\begin{array}{l}\text { Vicepresidente de Pequeño Derecho de SGAE. Miembro } \\
\text { del Consejo de Administración de la Sociedad de Artistas } \\
\text { Intérpretes o Ejecutantes de España (AIE) (1999-2012). }\end{array}$ \\
\hline & D. José Manuel Gamboa & $\begin{array}{l}\text { Periodista, escritor y productor musical español, } \\
\text { especialista en flamenco. Actualmente desempeña el cargo } \\
\text { en SGAE de analista técnico musical en la especialidad } \\
\text { de flamenco y es miembro del cuerpo académico de la } \\
\text { Cátedra de Flamencología de Jerez de la Frontera. }\end{array}$ \\
\hline \multirow{2}{*}{$\begin{array}{l}\text { Representantes de la Sociedad } \\
\text { de Artistas Intérpretes o } \\
\text { Ejecutantes de España (AIE) }\end{array}$} & D. Álvaro Hernández-Pizón & Director de Asesoría jurídica y Estrategia de Recaudación. \\
\hline & D. Pedro Rivas Prieto & Abogado de AIE. \\
\hline \multirow{3}{*}{ Gestores culturales } & D. Cristóbal Ortega Martos & Director artístico de la Bienal de Sevilla7 desde 2014. \\
\hline & $\begin{array}{l}\text { Dña. Isamay Benavente } \\
\text { Ferrera }\end{array}$ & Directora artística del Festival de Jerez de la Frontera ${ }^{8}$. \\
\hline & D. Manuel Herrera Rodas & $\begin{array}{l}\text { Actualmente es director artístico de los jueves flamencos } \\
\text { y miembro del Consejo Asesor de la Bienal de Sevilla. } \\
\text { Además fue director artístico de la Bienal de Sevilla en el } \\
\text { periodo } 1997-2006 \text {. }\end{array}$ \\
\hline $\begin{array}{l}\text { Centro Andaluz de } \\
\text { Documentación del Flamenco } \\
(\text { CADF })^{10}\end{array}$ & $\begin{array}{l}\text { Dra. Ana María Tenorio } \\
\text { Notario }\end{array}$ & $\begin{array}{l}\text { Responsable del Departamento de Documentación del } \\
\text { CADF. }\end{array}$ \\
\hline
\end{tabular}


1. Todos ellos son tanto intérpretes como compositores de buen número de obras.

2. Título honorífico creado por la Junta de Andalucía en 1985, que tiene por objeto reconocer "las acciones, servicios y méritos excepcionales o extraordinarios realizados en tiempos de paz por ciudadanos, grupos o entidades andaluces, españoles o extranjeros" que representen "el ejercicio de virtudes individuales o colectivas que tengan como referencia la solidaridad y el trabajo en beneficio de los demás ciudadanos" (decreto 117/85, de 5 de junio).

3. Medalla que concede el Ministerio de Cultura de España a aquellas personas o instituciones que destaquen en los campos literario, dramático, musical, coreográfico, de interpretación, etc.

4. Reconocimiento que otorga la Bienal de Sevilla.

5. Reconocimiento que otorga el Festival Internacional del Cante de las Minas, celebrado cada año en el municipio de La Unión (en la Región de Murcia).

6. Reconocimiento otorgado por el Concurso Nacional de Arte Flamenco de Córdoba, certamen de carácter trianual que se celebra en la ciudad de Córdoba (España) desde 1956.

\section{Anexo 2. Preguntas realizadas a los expertos entrevistados}

1. En su opinión ¿̇uáles son los principales elementos que forman la obra flamenca? De los elementos mencionados ¿cuáles provienen del intérprete?

2. En su concepto ¿qué elementos reflejan la originalidad/creatividad de la contribución del intérprete a la obra flamenca?

3. ¿Cómo se puede medir dicha contribución?

\section{Anexo 3. Preguntas utilizadas de la encuesta}

a) Por favor, indique el nivel de aporte [desde 1 hasta 7 , siendo 1 muy bajo o nulo y 7 muy alto] al proceso creativo de la obra musical flamenca del siguiente agente interviniente: el intérprete de la obra.

b) Por favor, indique el nivel de acuerdo/desacuerdo [desde 1 hasta 7 , siendo 1 muy bajo o nulo y 7 muy alto] con las siguientes afirmaciones:

\section{Anexo 4. Medios utilizados para escuchar flamenco}

La pregunta que se realizó en la encuesta fue: El medio que utiliza normalmente para escuchar flamenco es... Los resultados porcentuales de utilización de los distintos medios se recogen en la figura 5 .
7. La Bienal es el gran acontecimiento internacional del mundo del flamenco. Se celebra cada dos años en Sevilla, donde se dan cita los aficionados y profesionales del sector de todo el mundo. Para más información: http://www.labienal.com/

8. Jerez de la Frontera es considerado por los entendidos del flamenco como uno de los puntos geográficos más importantes. La ciudad tiene un estilo propio y una idiosincrasia muy definida, siendo su festival uno de los más importantes ofrecidos por el mercado. Para más información: http://www.jerez.es/ webs_municipales/festival_jerez/

9. Ciclo de conciertos y recitales de flamenco organizados por la Fundación Cajasol (https://fundacioncajasol.com/tag/juevesflamencos/).

10. EI CADF (http://www.juntadeandalucia.es/cultura/centroandaluzflamenco/) se crea en 1987 para la conservación del arte jondo a través de una importante labor de recuperación, catalogación y difusión del patrimonio cultural andaluz.
4. Por favor, explique con el mayor grado de detalle la metodología que utiliza para el desarrollo de su trabajo. Específicamente ¿cómo participa en el proceso de creación artística? Describa el momento de la puesta en escena.

5. ¿Qué importancia tiene la intuición y la improvisación en el flamenco?

1. El elemento que va a decidir en última instancia que la obra sea un éxito o un fracaso será el intérprete.

2. Cuando asiste a un concierto de flamenco basa su elección en el intérprete que va a actuar.

3. Considero esencial en el flamenco la aportación del intérprete puesto que le impone su estilo y distinción especial. 
Figura 5. Porcentaje de utilización de los medios de escucha

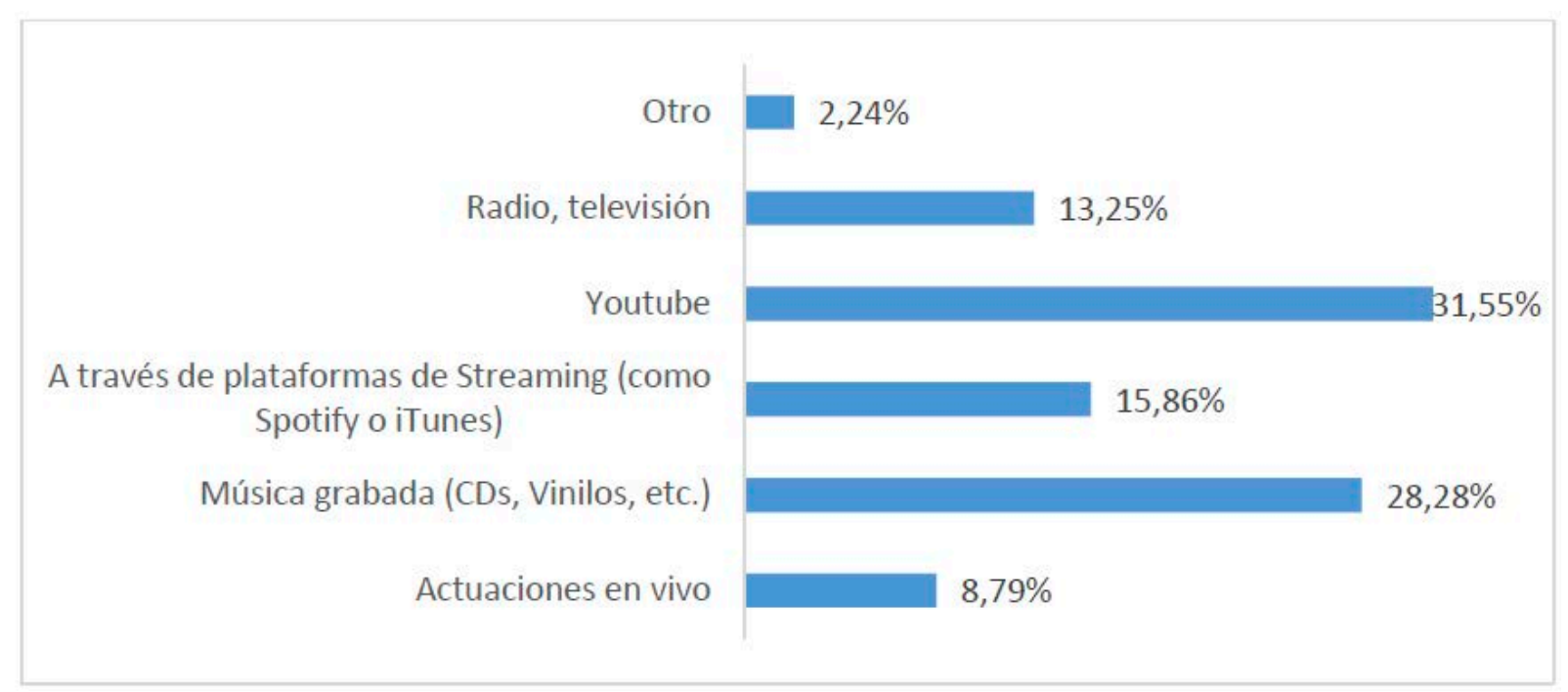

Fuente: Elaboración propia. 